\title{
Succinate dehydrogenase 5 regulates lung cancer metastasis by reprogramming glucose metabolism
}

\author{
Jie $\mathrm{Li}^{1{ }^{1 *}}$, Zhan Tuo ${ }^{1 *}$, Yan Zong ${ }^{1}$, Jun Liu ${ }^{2}$ \\ ${ }^{1}$ Cancer Center, Union Hospital, Tongji Medical College, Huazhong University of Science and Technology, Wuhan, China; ${ }^{2}$ Department of \\ Thoracic Surgery, Zhongnan Hospital of Wuhan University, Wuhan, China \\ Contributions: (I) Conception and design: J Liu; (II) Administrative support: J Liu; (III) Provision of study materials or patients: Y Zong; (IV) \\ Collection and assembly of data: J Li; (V) Data analysis and interpretation: Z Tuo; (VI) Manuscript writing: All authors; (VII) Final approval of \\ manuscript: All authors. \\ "These authors contributed equally to this work and should be considered as co-first authors. \\ Correspondence to: Jun Liu. Department of Thoracic Surgery, Zhongnan Hospital of Wuhan University, Wuhan 430000, China. \\ Email: liujun0129@hust.edu.cn.
}

Background: Lung cancer is the leading cause of cancer-related death globally, with many of these patients also suffering from diabetes. Previous studies have shown that diabetes may contribute to cancer progression through hyperglycemia. However, the underlying mechanism remains largely unknown. This study aimed to investigate the role of succinate dehydrogenase 5 (SDH5), an enzyme required for assembling respiratory complex II in lung cancer patients with diabetes.

Methods: The expression levels of SDH5 in patient plasma and tissue were determined by RT-qPCR. Western blotting, immunofluorescence (IF), and immunohistology (IHC) were used to examine protein expression. Migration and invasion assays were performed using Transwell assays. Reactive oxygen species (ROS) production was detected by flow cytometry. Bioluminescent imaging (BLI) was used to detect tumor metastasis in a lung orthotopic mouse model.

Results: In samples from non-small cell lung cancer (NSCLC) patients with diabetes, SDH5 mRNA levels were significantly lower in both plasma and tissue among later stage patients. TCGA data showed that low SDH5 expression was correlated with a higher expression of genes involved in glycolysis and metastasis. In vitro, high glucose conditions alone induced epithelial-to-mesenchymal transition (EMT) in cells, an effect that was further reinforced by SDH5 depletion. Additionally, depleting SDH5 promoted glucose consumption and lactate production. The underlying mechanism indicates that depleting SDH5 stabilizes hypoxia-inducible factor 1-alpha (HIF-1 $\alpha$ ), which is dependent on ROS production. In vivo, SDH5-deficient tumor-bearing mice had multiple organ metastases, which is consistent with the in vitro findings.

Conclusions: Our findings suggest that SDH5 deficiency activates HIF-1 $\alpha$ to promote EMT under high glucose conditions and represents a predictive marker for NSCLC patients with diabetes.

Keywords: Non-small cell lung cancer (NSCLC); high glucose; succinate dehydrogenase 5 (SDH5); epithelial-tomesenchymal transition (EMT); hypoxia-inducible factor 1-alpha (HIF-1 $\alpha$ )

Submitted Oct 26, 2021. Accepted for publication Nov 23, 2021.

doi: $10.21037 /$ jtd-21-1769

View this article at: https://dx.doi.org/10.21037/jtd-21-1769

\section{Introduction}

Lung cancer is the leading cause of cancer-related death worldwide, and approximately $80 \%$ of these patients have non-small cell lung cancer (NSCLC). Epidemiological evidence suggests that individuals with diabetes have a higher risk of different cancer types that may be mediated by hyperglycemia $(1,2)$. However, relationship between cancer metabolism and cancer progression are still largely unclear. 
Succinate dehydrogenase 5 (SDH5) plays an essential role in the assembly of succinate dehydrogenase (SDH), also referred to as respiratory complex II (3). Thus, SDH5 is required for an intact Krebs cycle. Previous studies have found that mutated SDH5 increases the risk of initiation and progression of several cancers, including paraganglioma and gastrointestinal stromal tumors $(4,5)$. Our previous studies have demonstrated that SDH5 regulates glycogen synthase kinase $3 \beta$ - $\beta$-catenin-mediated lung cancer metastasis and plays a suppressive role in $\mathrm{CD} 8^{+} \mathrm{T}$ cells $(6,7)$. However, few studies have investigated SDH5 in cancer metabolism. Considering metabolic status of cancer patients with diabetes and the fact that SDH5 is a key component of both the Krebs cycle and mitochondrial electron transport chain, we aimed to determine whether SDH5 is involved in the tumor progression of NSCLC patients with diabetes.

A key characteristic feature of epithelial-to-mesenchymal transition (EMT) is cell morphology and decreasing E-cadherin expression or upregulating vimentin expression participates in this process (8). A previous study showed that hypoxia-inducible factor 1 -alpha (HIF-1 $\alpha$ ) directly induces EMT by activating the TGF- $\beta$ signaling pathway (9). In the present study, we investigated the role of SDH5 in NSCLC with diabetes in vivo and in vitro. Patients with low SDH5 expression had poor outcomes, and the cancer genome atlas (TCGA) data further confirmed that lower SDH5 expression correlates with higher expression of genes involved in metastasis and glycolysis. In vitro, depleting SDH5 promoted EMT by stabilizing HIF-1 $\alpha$ expression, which is dependent on reactive oxygen species (ROS). We confirmed these findings in vivo using a xenograft mouse model. We conducted a competing endogenous RNA (ceRNA) network and identify the $\operatorname{lncRNA} / \mathrm{miR}-23 \mathrm{c} / \mathrm{SDH} 5$ regulatory axis as an important player in NSCLC progression. Our findings provide a new mechanism of SDH5 in tumor metastasis in NSCLC patients with diabetes. More importantly, it can be achievable testing SDH5 mRNA from patients' plasma, indicating a potential clinical translation by using SDH5 as a liquid biopsy marker to track cancer progression

We present the following article in accordance with the ARRIVE reporting checklist (available at https://dx.doi. org/10.21037/jtd-21-1769).

\section{Methods}

\section{Patient samples}

We initiated an open, analytical, observational, and retrospective study of 30 patients from May 2017 to October 2017. The patients had been diagnosed with stage I and III NSCLC and had a medical history of diabetes. Importantly, the patients had poor control of their blood sugar levels, showing random plasma glucose levels $\geq 200 \mathrm{mg} / \mathrm{dL}$ for two times. All procedures performed in this study involving human participants were in accordance with the Declaration of Helsinki (as revised in 2013). The study was approved by The Ethical Committee of Huazhong University of Science and Technology and informed consent was taken from all the patients. The study was registered at Chinese Clinical Trial Registry (ChiCTR1800014878). Thirty lung cancer tissues were retrieved by experienced surgical pathologists from Wuhan Union Hospital using computed tomography (CT)-guided needle biopsy. For each patient, $5 \mathrm{ml}$ of blood was collected before treatment in tubes containing ethylene diamine tetraacetic acid (EDTA), and the fresh tumors and blood samples were kept at $4{ }^{\circ} \mathrm{C}$ and sent to the laboratory within 2 hours of collection. The characteristics of all patients are shown in Table S1.

\section{mRNA expression in patient blood and tissues}

Blood samples were processed within 2 hours of collection by centrifugation at $4,000 \mathrm{rpm}$ for $30 \mathrm{~min}$ at $4{ }^{\circ} \mathrm{C}$. In total, $500 \mu \mathrm{L}$ of plasma from each sample was extracted and subjected to RNA extraction according to the manufacturer's instructions.

\section{Cell culture}

Lung cancer cell lines (A549, HCC827, H522, and H460) were purchased from the Chinese Academy of Science Committee on Type Culture Cell Bank (Shanghai, China). All the experiments were performed under high glucose conditions, in order to mimic hyperglycemic $(200 \mathrm{mg} / \mathrm{dL})$ conditions. Glucose was purchased from Sigma (G8270100G, St. Louis, USA). The cells were cultured in highglucose medium containing $500 \mathrm{~mL}$ of RPMI 1640 medium, no glucose (Gibco, 11879020, Waltham, Massachusetts, USA), $100 \mathrm{mg}$ of glucose, 10\% fetal bovine serum (FBS, Invitrogen, 10437, California, USA), and 1\% penicillin-streptomycin solution.

\section{SiRNA transfection and lentiviral infection (RNA silencing by siRNA and shRNA)}

Human SDH5 siRNA and shRNA were purchased from 
Santa Cruz (sc-96879 and sc-96879-SH). When monolayer cells reached $50 \%$ confluency, the cells were transfected with siRNA or shRNA according to the manufacturer's instructions.

\section{Quantitative RT-qPCR}

Total RNA was isolated from cells and tissues using TRIzol reagent (Invitrogen). cDNA was synthesized from total RNA using SYBR ${ }^{\circledR}$ Premix Ex TaqTM II (Takara Bio, Japan) according to the manufacturer's instructions. The sequences of genes were showed in Table S2.

\section{Western blotting analysis and antibodies}

Antibodies against SDH5, HIF-1 $\alpha$, E-cadherin, Vimentin, Prdx3, SOD1, GAPDH, Lamin A, and Actin were obtained from CST (Western Blot: 1:1,000), and anti-mouse or antirabbit secondary antibodies were used based on the isotype species of the primary antibody $(1: 3,000)$. The detailed protocol is described in our previous study (10).

\section{Fluorescence microscopy}

The detailed protocol is described in our previous study (6).

\section{In vitro migration and invasion assay}

Cancer cells $\left(5 \times 10^{4}\right.$ cells $)$ in $200 \mu \mathrm{L}$ of high glucose RPMI 1640 plus $0.1 \%$ FBS were first seeded into the upper chamber of a Transwell (Corning, Costar, NY, USA) coated with or without ECM gel (Sigma-Aldrich). The lower chamber was filled with $600 \mu \mathrm{L}$ of high glucose RPMI with $20 \%$ FBS. The detailed protocol is described in our previous study (6).

\section{Glucose consumption and lactate production assays}

To assess glucose consumption and lactate production, the cells $\left(2.5 \times 10^{5}\right)$ were cultured in complete medium. The supernatant was collected and assessed using a lactic acid assay kit (Nanjing Jiancheng Bio., Nanjing, China) according to the manufacturer's protocol.

\section{Flow cytometry analysis of ROS}

A549 and HCC 827 cells were transfected with siRNAs and harvested and washed three times with phosphate- buffered saline (PBS). The cells were then incubated with high glucose RPMI 1640 containing 10\% FBS and $25 \mathrm{mM}$ of 2',7'-dichlorofluorescein diacetate (DCF, Sigma) for $20 \mathrm{~min}$, and then collected. ROS levels were measured by BD FACS Calibur 2 Laser, 4 Color Flow Cytometer.

\section{Immunobistochemical analysis}

The detailed protocol is described in our previous study (10).

\section{Competing endogenous $R \mathrm{NA}$ network construction}

To illustrate the potential capabilities of SDH5 in LUAD, miRTarBase (http://mirtarbase.cuhk.edu.cn/), StarBase (http://starbase.sysu.edu.cn/) and LncBase Predicted v.2 (https://carolina.imis.athena-innovation.gr/diana_tools/ web/index.php? $r=\operatorname{lncbasev} 2 /$ index-predicted) were used to predict miRNA and lncRNA targets. We also used the TCGA dataset to explore the expression and prognostic value of these miRNA and lncRNA targets. Results were considered statistically significant when $\mathrm{P}<0.05$.

\section{Animal experiments}

Six-week-old male nude mice (Beijing HFK Bioscience, China) were housed under pathogen-free conditions according to the animal care guidelines of Huazhong University of Science and Technology (HUST). Animal experiments were performed under a project license (No. SCXK (HUBEI) 2016-0009) granted by Animal Experimentation Ethics Committee of Huazhong University of Science and Technology (HUST, Wuhan, China), in compliance with Hubei Provincial Animal Care and Use Committee guidelines for the care and use of animals. Diabetes was induced in nude mice by administering clinical grade STZ (Zanosar, Sicor Pharmaceuticals, Irvine, CA, USA). Heavier mice (weighing more than $25 \mathrm{~g}$ ) were selected preferentially because they are most suitable for STZ induction because of potential weight loss after induction. Pre-chilled STZ was delivered intraperitoneally at a dose of $240 \mathrm{mg} / \mathrm{kg}$ to induce diabetes. The day after induction, $1 \mathrm{~mL}$ of saline was injected subcutaneously daily. Blood glucose levels were measured by bleeding the tail vein on days 3 and 4 after induction. Mice with a blood glucose level $>200 \mathrm{mg} / \mathrm{dL}$ for 2 consecutive days were considered diabetic, at which time injection was initiated. Transfected shRNA control and shRNA SDH5 cells $\left(5 \times 10^{5} / 20 \mu \mathrm{L}\right.$ in PBS) were mixed with Matrigel at a 1:1 ratio. 
For surgery, the first 4-mm incision was made on the left lateral chest. Next, the soft fat tissue was carefully removed to expose the thoracic ribs and intercostal space, allowing for better visualization of the left lobe of the lung. During surgery, the cell mix was injected directly into the lungs of 5 -week-old male nude mice in the two groups $(n=5)$. Surgical staples were removed 1 week after injection. The weight of all the mice was measured three times per week. Twenty-five days after injection, Bioluminescence imaging (BLI) was performed using an IVIS Imaging System (Xenogen). Briefly, mice were anesthetized, injected with D-luciferin $(150 \mathrm{mg} / \mathrm{kg}$, intraperitoneally), and imaged 2 $\mathrm{min}$ after injection for $10 \mathrm{~min}$.

\section{Statistical analysis}

For in vitro data, each experiment required three replicates in three independent experiments. The results were presented as means \pm SE (standard error). An unpaired t-test and one-way ANOVA (Analysis of Variance) were used for multiple group analysis with parametric data. Flow cytometry data were analyzed using FlowJo (Version 7.6.5), and all calculations were performed using GraphPad Prism (v. 8.0). A $\mathrm{P}$ value $<0.05$ was considered statistically significant.

\section{Results}

\section{Loss of SDH5 predicts a poor outcome in NSCLC patients with diabetes}

Specimens from lung cancer patients were obtained via CTguided percutaneous needle biopsy (Figure $1 A$ ). SDH5 was mainly located in the cytoplasm (Figure 1B). The Spearman rank correlation test showed that the SDH5 mRNA levels were positive correlated $($ rho $=0.943 ; \mathrm{P}<0.01)$ between patient plasma and tumors (Figure 1C). To investigate the role of SDH5 in patient outcomes, we compared the SDH5 expression levels in patients with different clinical stages. As shown in Figure 1D,1E, patients with stage I disease had higher mRNA levels of SDH5 than those with stage III disease, indicating that lower SDH5 expression may be correlated with poor outcomes in NSCLC lung cancer patients with diabetes.

Next, we downloaded data from TCGA database to examine the expression of SDH5 and genes that are commonly associated with metastasis and glucose metabolism. As shown in Figure $1 F$, the heat map results demonstrated that patients with low SDH5 expression had higher expression levels of glycolytic-related genes (HIF1A, $A C L Y, A C A C A$, and HK1) and metastasis-associated genes (Vim, CTNNB1, mTOR, and PIK3CA), which supported our finding that patients in higher stages have less SDH5. More importantly, gene set enrichment analysis (GSEA) showed that SDH5 was significantly negatively correlated with type II diabetes and positively correlated with oxidative phosphorylation (Figure 1G,1H), indicating that diabetes may negatively regulate SDH5 and that SDH5 deficiency may impair oxidative phosphorylation.

\section{SDH5 deficiency reinforces high glucose induced EMT in NSCLC cells}

To uncover the underlying mechanism, we first selected four NSCLC cell lines (H522, H460, HCC827, and A549) in vitro to detect the baseline expression of SDH5. SDH5 was highly expressed in A549 and HCC827 cell lines (Figure 2A); thus, we used these two cell lines for subsequent experiments. Since hyperglycemia is a key feature of diabetes, we cultured the cells with high glucose medium for 48 hours. Both cell lines changed their morphology, similar to the phenotypic changes observed during EMT (Figure 2B). Our previously published study showed that SDH5 regulates the EMT process (6); therefore, we first evaluated EMT-related proteins under high glucose culture conditions. The expression of the cell-cell junction protein E-cadherin was decreased, while that of the vimentin filament and fibronectin proteins vimentin and $\mathrm{N}$-cadherin was increased, respectively (Figure 2C).

The above results showed that hyperglycemia alone activates the EMT process. Thus, we speculated about the role of SDH5 in this process. After knocking down SDH5, E-cadherin expression was significantly reduced, and the $\mathrm{N}$-cadherin and vimentin protein levels were much higher than those in the control group (Figure 2D,2E). Taken together, these results indicate that SDH5 downregulation may exert a superimposed effect that promotes the process of high-glucose-induced EMT.

\section{Loss of SDHS promotes metastasis and glycolysis in NSCLC cells}

The first step in cancer cell metastasis is gaining the ability of migration and invasion. After knocking down SDH5, SDH5-deficient cancer cells showed significantly increased migration and invasion after $24 \mathrm{~h}$ (Figure $2 F, 2 G$ ). Our 
A

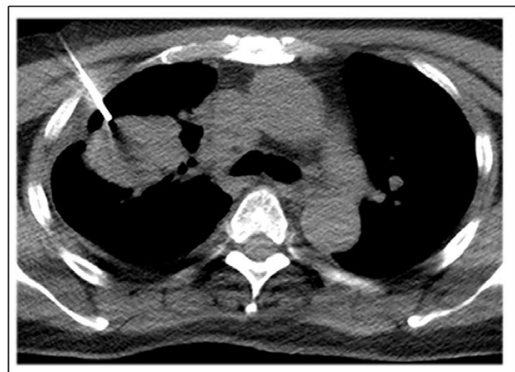

B

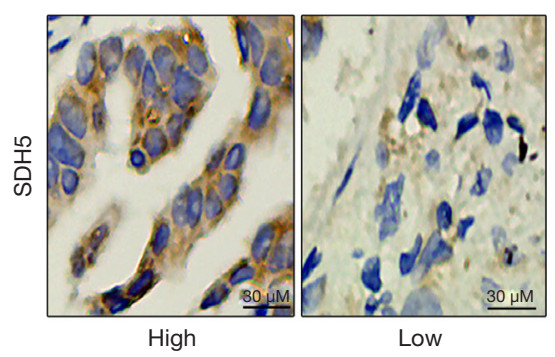

D

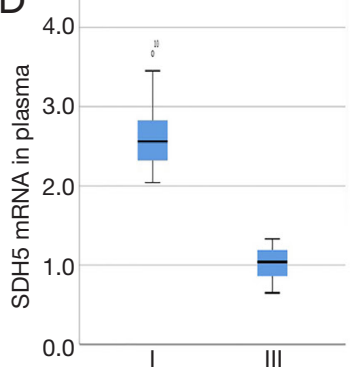

F

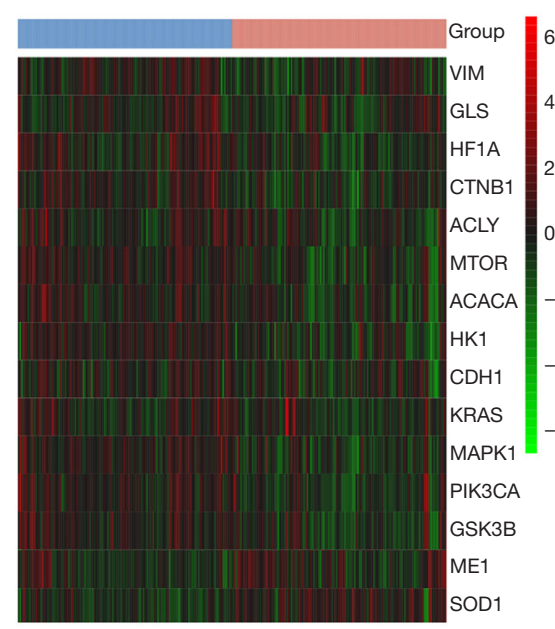

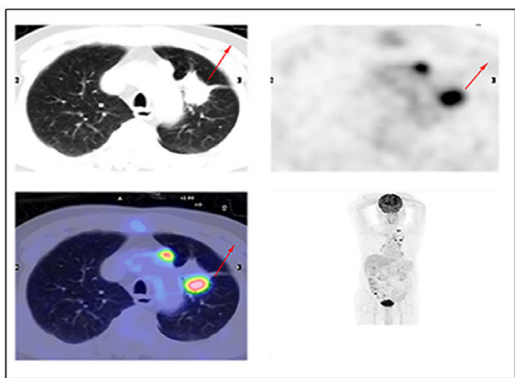

C
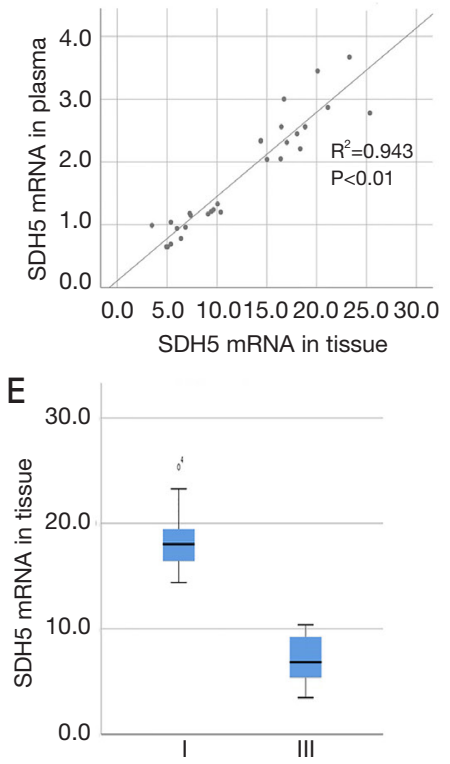

G Enrichment plot: KEGG_TYPE_II_DIABETES_MELLITUS

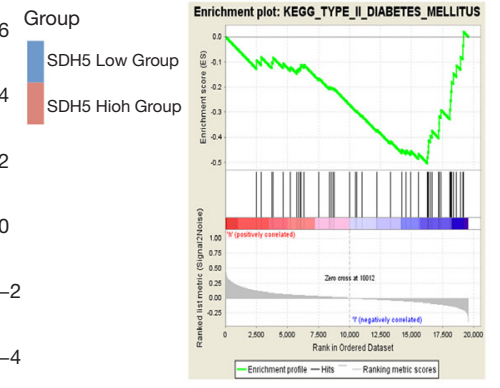

$\mathrm{H}$

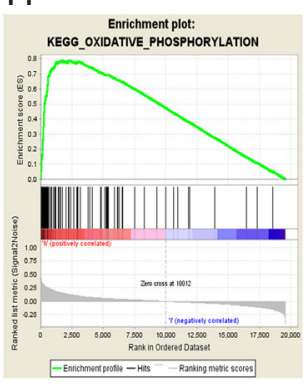

Figure 1 Expression of SDH5 in patient samples. (A) Thirty lung cancer specimens were retrieved by experienced surgical pathologists via CT-guided needle biopsy (the red arrow shows the insertion position of the puncture needle); (B) SDH5 expression as assessed by IHC; (C) Correlations between plasma and tissue mRNA expression levels of SDH5; (D,E) Correlation between clinical stages and plasma/tissue expression levels of SDH5. The lines inside the boxes denote the medians. The whiskers of the box plots represent the SE, 95\% CI; (F) TCGA heat map analysis of differences between the expression of SDH5 and metabolic-related genes; (G) GSEA of SDH5 and the diabetes pathway $(\mathrm{P}<0.05)$; $(\mathrm{H})$ GSEA of SDH5 and the oxidative phosphorylation pathway $(\mathrm{P}<0.05)$. SDH5, succinate dehydrogenase 5; IHC, immunohistology. 
previous study revealed that SDH5 mediates lung cancer metastasis by regulating the glycogen synthase kinase $3 \beta-$ $\beta$-Catenin signaling pathway. Therefore, we first verified whether this pathway was activated under high glucose metabolism. Interestingly, no significant change was found in this pathway after depleting SDH5 (Figure 2H). Previous studies have shown that high glucose alone induces Ser9 phosphorylation and inactivates GSK $3 \beta$, possibly explaining why the role of SDH5 in the GSK $3 \beta-\beta$ Catenin signaling pathway is compensated by high glucose-induced inactivation (11). However, we observed that under high glucose conditions, depleting SDH5 still promoted EMT.

Considering the specificity of the role of SDH5 in glucose metabolism (Figure 1G,1H), we next tested changes in the levels of glucose metabolites. After depleting SDH5, the cells showed significantly higher glucose consumption and lactic acid production, indicating that they shifted their metabolic status to increased glycolysis to satisfy their energy needs when they had impaired tricarboxylic acid
(TCA) (Figure 2I,2f). Taken together, these results indicate that under high glucose conditions, SDH5 may activate another signaling pathway to promote NSCLC cell line metastasis, possibly correlated with its role in glycolysis.

\section{Loss of SDH5 stabilizes HIF-1a expression under high glucose conditions}

Cancer cells favor glycolysis not only due to the rapid energy demand, but also because they adapt when they are stuck in a harsh hypoxic tumor microenvironment (TME). HIF- $1 \alpha$ is a key factor in both low-pH and hypoxic TMEs (12). Therefore, we investigated whether HIF-1 $\alpha$ participates in the process of glycolysis mediated by SDH5 and two classical metabolism-related pathways (mTOR and PI3K-AKT). However, we did not observe changes in these two pathways (data not shown). As expected, the protein level of HIF-1 $\alpha$ significantly increased after knocking down SDH5 and the two direct downstream targets, LDHA and
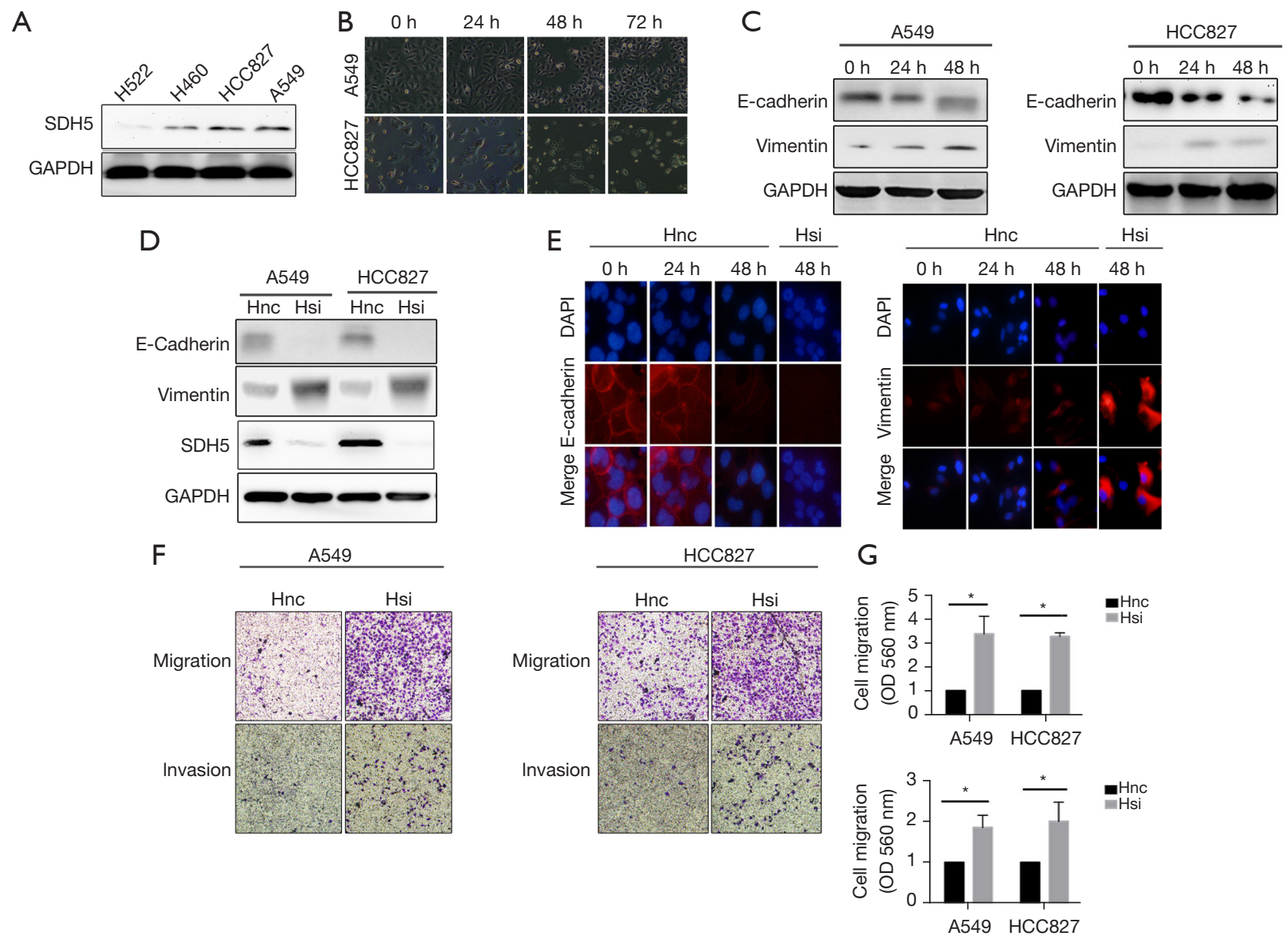

(c) Journal of Thoracic Disease. All rights reserved. 

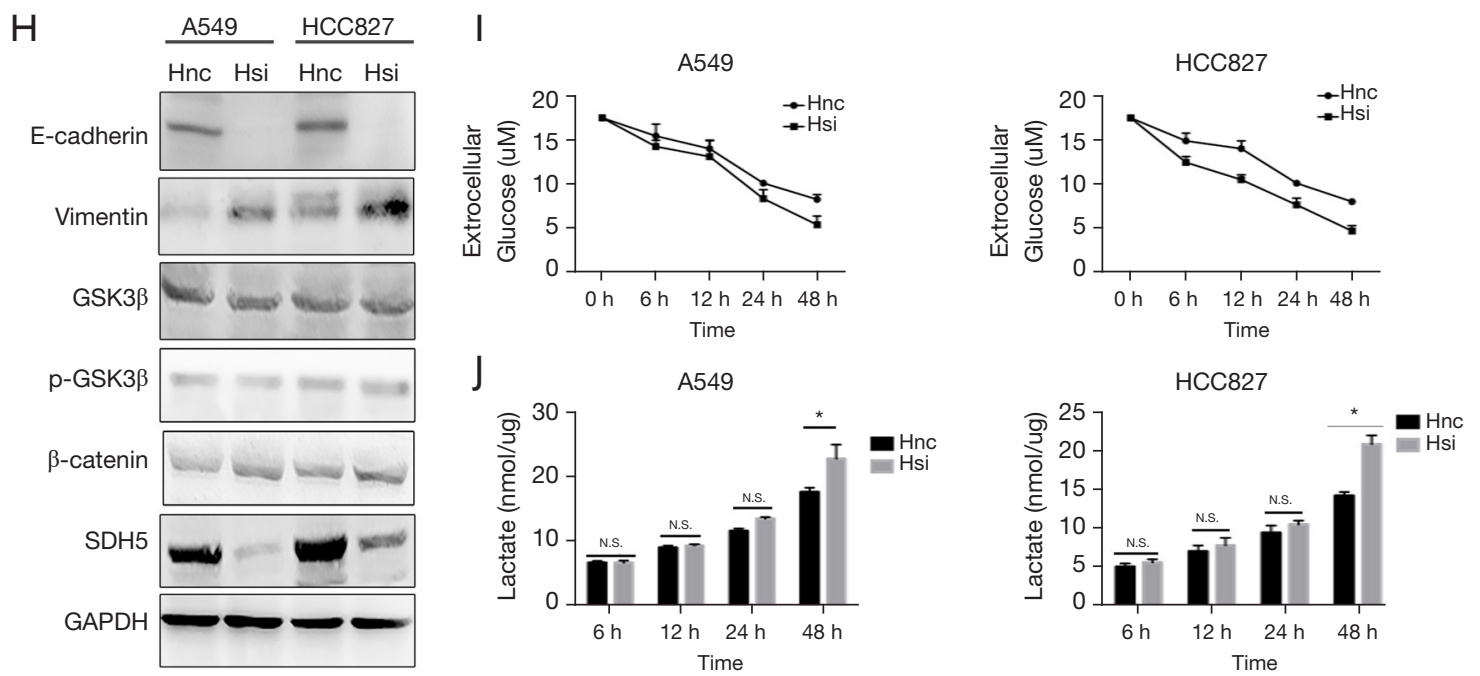

Figure 2 SDH5 depletion enhances the metastasis and glycolysis of NSCLC cells. (A) SDH5 expression in difference NSCLC cell lines; (B) Morphology of A549 and HCC827 cells (magnification, x40); The cell was observed by phase-contrast microscopy. (C) Protein levels of E-cadherin and vimentin; (D) Protein levels of E-cadherin and Vimentin in SDH5 knockdown and control cells; (E) E-cadherin and Vimentin expression levels in SDH5 knockdown and control cells as assessed by immunofluorescence (magnification, $\times 400$ ); Cells were incubated with primary antibody $(1: 200)$ overnight at $4{ }^{\circ} \mathrm{C}$. Secondary antibody (anti-rabbit Alexia fluor 555) were used to incubate in $1: 500$ for 2 hours at room temperature, and then slides were wash with PBS for three times and finally incubated with DAPI in 1:5,000 ratio for 5 min. (F) Migration in Transwell chambers for $48 \mathrm{~h}$; The cells on the lower surface of the membrane were stained with Cell Stain (Chemicon, Tokyo, Japan) and quantified by measuring absorbance at 560. (G) Quantitative measurements of migrating cells were determined. The data are presented as means $\pm \mathrm{SE}$ (error bars; $\mathrm{P}<0.01$ ). Each experiment was performed twice, with triplicates for each assay; the scale bar was $100 \mu \mathrm{m}$. (H) Protein expression of the GSK3 $\beta$ - $\beta$-Catenin signaling pathway; (I) Extracellular glucose assay at different timepoints with triplicates for each assay; (J) Lactate assay with triplicates for each assay. * $\mathrm{P}<0.05$; ns, $\mathrm{P}>0.05$. SDH5, succinate dehydrogenase 5; NSCLC, non-small cell lung cancer.

\section{PDK1 (Figure 3A).}

Additionally, the transcriptional level of HIF-1 $\alpha$ did not change, indicating that SDH5 may affect HIF- $1 \alpha$ protein stability (Figure 3B). To verify this hypothesis, we used MG132, a proteasome inhibitor, to inhibit protein degradation. The effect of SDH5 on HIF-1 $\alpha$ upregulation was abolished, and both groups showed similar expression levels of HIF-1 $\alpha$. Interestingly, for the A549 cell line, slightly higher HIF-1a expression was observed in the SDH5-knockdown group, indicating that loss of SDH5 upregulates HIF-1 $\alpha$ mainly by interfering with protein stabilization (Figure 3C).

\section{SDHS is dependent on ROS to stabilize HIF-1a}

ROS play a crucial role in stabilizing HIF-1 $\alpha$, and previous studies have reported that diabetes induces and generates ROS (13-15). Therefore, we first determined whether SDH5 is dependent on ROS to stabilize HIF- $1 \alpha$. As expected, depleting SDH5 in both A549 and HCC827 cells resulted in higher ROS levels (Figure 3D). Next, we tested whether suppressing ROS blocked SDH5-mediated HIF-1 $\alpha$ stabilization. Treatment with the ROS inhibitor scavenger $\mathrm{N}$-acetylcysteine (NAC) abolished the effect of SDH5 depletion activating HIF-1 $\alpha$ (Figure $3 E$ ), indicating that SDH5 regulates HIF- $1 \alpha$ through ROS in cancer cells.

Next, we measured the levels of antioxidant enzymes [peroxiredoxin $3(\operatorname{Prdx} 3)$ and mitochondrial superoxide dismutase 2 (SOD1)], which are involved in the catalytic transformation of ROS and are directly associated with ROS production (16). Loss of SDH5 deregulated the expression of SOD1 and Prdx3 (Figure 3F). Since nuclear factor erythroid 2-related Factor 2 (Nrf2) controls the gene transcription of antioxidant enzymes, we evaluated its activation (17). Interestingly, depleting SDH5 inhibited Nrf2 nuclear translocation (Figure $3 G, 3 H$ ), leading to reduced SOD1 mRNA levels (Figure 3I). Taken together, our data showed that SDH5-mediated stabilization of HIF- 
A

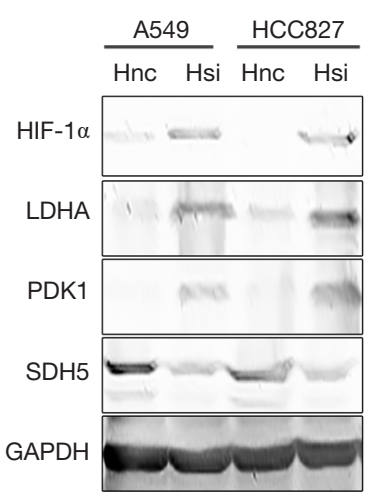

D
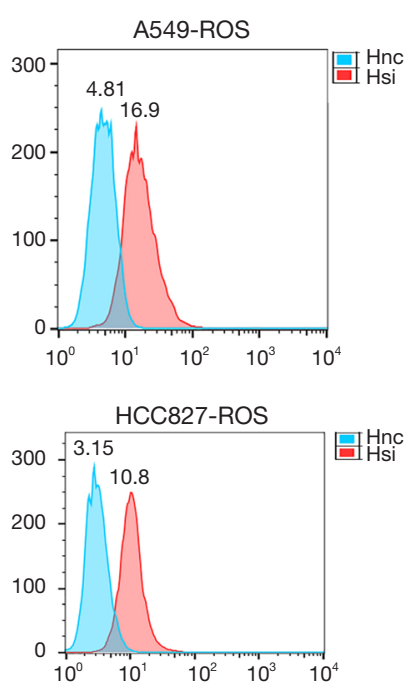

G

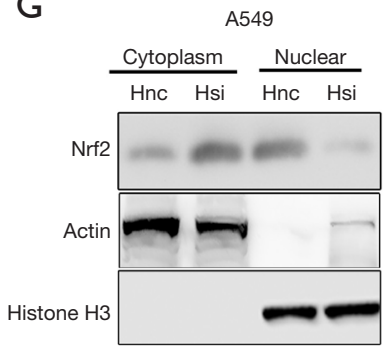

B

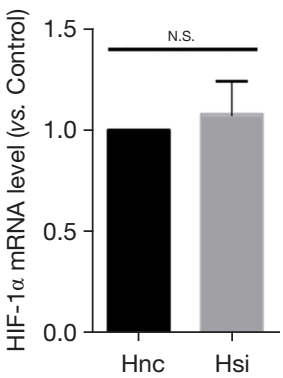

E

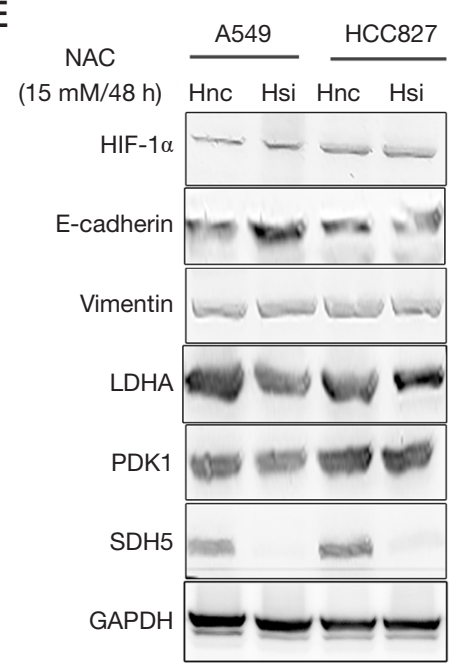

$\mathrm{H}$

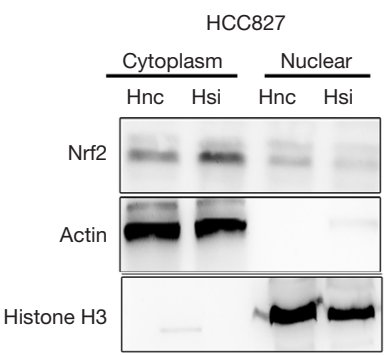

C

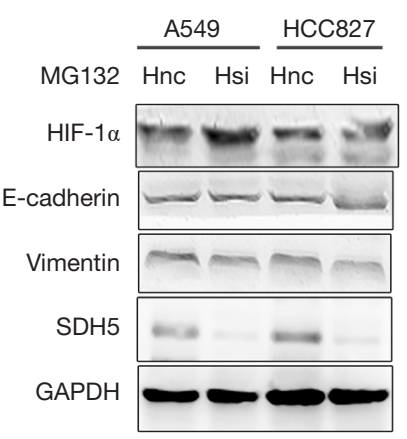

F

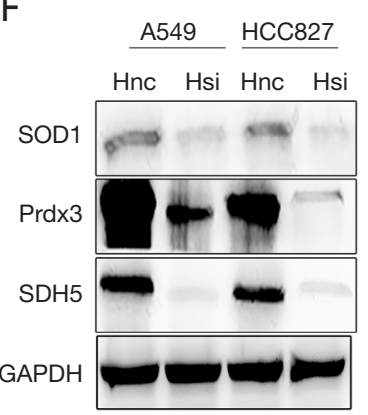

Figure 3 Loss of SDH5 stabilizes HIF-1 $\alpha$ in a ROS-dependent manner. (A) Protein levels of HIF-1 $\alpha$ and its downstream targets; (B) HIF$1 \alpha$ mRNA levels. The relative mRNA levels of HIF-1 $\alpha$ were quantified by $2{ }_{\Delta \Delta} \mathrm{CT}\left({ }_{\Delta} \mathrm{CT}=\mathrm{CT}{ }^{\mathrm{HIF}-1 \alpha}-\mathrm{CT}^{\mathrm{GAPDH}},{ }_{{ }_{\Delta}} \mathrm{CT}={ }_{\Delta} \mathrm{CT}\right.$ SDH $5 \mathrm{si}_{\Delta} \mathrm{CT}$ SDH5 control); (C) Protein levels of HIF-1 $\alpha$, E-cadherin, vimentin, and SDH5 after incubation with MG132 (20 $\mu$ M) for 1 hour. (D) ROS production by flow cytometry; (E) Protein levels of HIF-1 $\alpha$, E-cadherin, vimentin, LDHA, and PDK1 after treatment with NAC (10 mM) for 24 h; (F) Protein levels of SOD1 and Prdx3; (G,H) Nuclear translocation of Nrf2 was evaluated using cytosolic and nuclear fractions. Histone $\mathrm{H} 3$ and actin were used as loading controls; (I) mRNA levels of SOD1 as assessed by RT-qPCR. *, P<0.05. SDH5, succinate dehydrogenase 5; HIF-1 $\alpha$, hypoxia-inducible factor 1-alpha; ROS, reactive oxygen species; NAC, N-acetylcysteine. 
$1 \alpha$ is ROS dependent.

To clarify the potential molecular mechanism of SDH5 in LUAD, we constructed an mRNA-miRNA-lncRNA interaction network. We identified 4 miRNAs (miR-485$5 \mathrm{p} / \mathrm{miR}-23 \mathrm{c} / \mathrm{miR}-16-5 \mathrm{p} / \mathrm{miR}-1286$ ) as target miRNAs bound to SDH5 according to miRTarBase and starBase (Figure S1A). Among them, hsa-miR-23c demonstrated the highest target score according to the CLIP Data; we therefore explored its upstream lncRNA target to construct the miRNA-lncRNA axis. Four lncRNAs (ZNF674-AS1/XIST/TYMSOS/NEAT1) were identified as target LncRNAs (Figure S1B). A ceRNA network was visualized; ZNF674-AS1 and SDH5 were positively correlated (Figure S1C). Survival analysis demonstrated that high ZNF674-AS1 were associated with better prognosis (Figure S1D).

\section{Depleting SDHS enhances metastasis in a xenograft mouse model with diabetes}

To verify the above results in vivo, we first generated diabetic nude mice by STZ induction. After successfully establishing a diabetes model, the mice were divided into two groups. Mice bearing SDH5-deficient tumors showed more metastasis (Figure $4 A, 4 B$ ). Importantly, cancer cells could only be detected in the lymph nodes and liver in the HE slides of the SDH5-deficient group (Figure 4C). Additionally, by IHC, E-cadherin expression was found to be significantly downregulated and vimentin expression was increased in those organs, and these findings were also consistent with our in vitro results (Figure 4D). Taken together, depleting SDH5 in NSCLC tumors enhances metastasis in a xenograft mouse model with diabetes.

\section{Discussion}

Cancer cells have unique metabolism profiles in TME which they prefer glycolysis rather than oxidative phosphorylation (OXPHOS) even exposing abundant oxygen (i.e. the Warburg effect). The rational of favoring glycolysis may be due to their huge demands of ATP and intermediate metabolites in a short time to support proliferation and progression of cancer cells. Glycolysis is not an economic way to generate ATP, however compared with OXPHOS, it is faster and generate lots of metabolite intermediates. To do this, cancer cells tend to impair their mitochondria function by key genes mutation in tricarboxylic acid (TCA) cycle as well as upregulate genes related glycolysis (HK2,
$P K M 2, L D H A)$ to maintain their glycolysis feature. Because of the high consumption of glucose in glycolysis, positron emission tomography (PET) with a radiolabeled analog of glucose (18F-fluorodeoxyglucose, FDG) has been widely used in clinical practice to detect tumor. To date, various reagents have been tested for targeting glucose metabolism in cancer cells, such as targeting hexokinase (2-DG, Lonidamine), pyruvate kinase-M2 (Shikonin, Oleanolic acid) (18). However, compared to detection and treatment, whether it is applicable to use cancer glucose related markers to predict the pathologic differentiation and stages is still largely uninvestigated.

In recent decades, diabetes, a chronic disease which closely correlated with rapid economic development, has become more and more common. A high proportion of newly diagnosed cancer patients have diabetes in their past medical history. Epidemiological evidence suggests that cancer patients with diabetes have a significantly poorer cancer treatment response and prognosis than non-diabetic cancer patients $(1,2)$. Thus, diabetes is closely related to the occurrence and progression of malignant tumors. Previous studies have suggested that this causality may be correlated with diabetes-specific features such as hyperglycemia. However, the underlying mechanism remains unclear.

$\mathrm{SDH}$ acts as an oxidative phosphorylation and respiratory chain electron transfer hub. In the enzyme complex, SDH5 is required to assemble SDH (19). Previous studies have shown that SDH5 mutation is closely related to the occurrence and development of several tumors $(4-6,10)$. One explanation is that a connection exists between SDH5 and the Warburg effect, given that SDH5 is a required enzyme in the Krebs cycle (3). Based on the role of SDH5 in tumor development and glucose metabolism, our study investigated whether SDH5 plays a role in progression in NSCLC patients with diabetes and whether it is associated with glucose metabolism.

We first found that under diabetic conditions, NSCLC patients with later-stage NSCLC and stage III NSCLC showed lower SDH5 expression (Figure 1A-1E). TCGA data also showed that patients with low SDH5 expression had higher expression levels of metastasis-associated genes and glycolytic-related genes (Figure $1 F-1 H$ ), a finding that is consistent with the GSEA data showing that SDH5 is negatively correlated with type II diabetes but is positively correlated with oxidative phosphorylation.

To explore the mechanism, we cultured cells in high glucose medium to better the mimic clinical settings in vitro. Surprisingly, high glucose alone changed the cell phenotype 
A
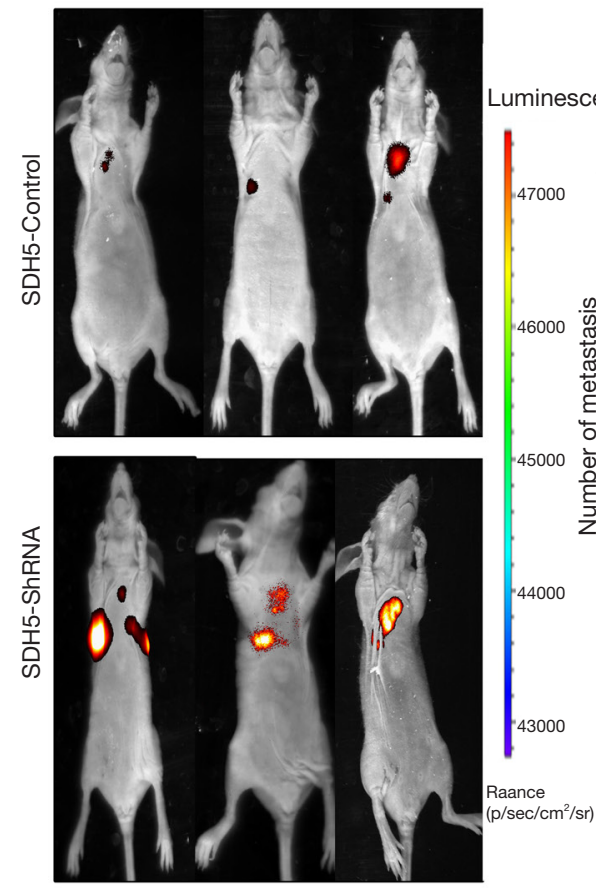

D

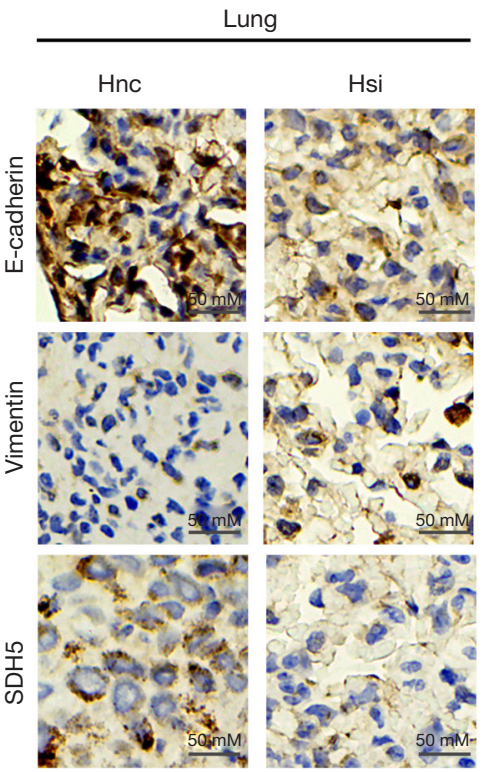

B

Luminescence

47000

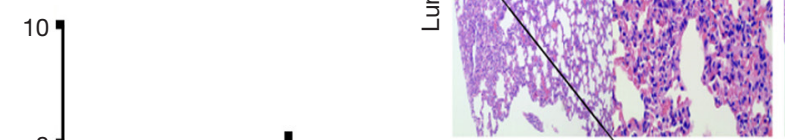

I

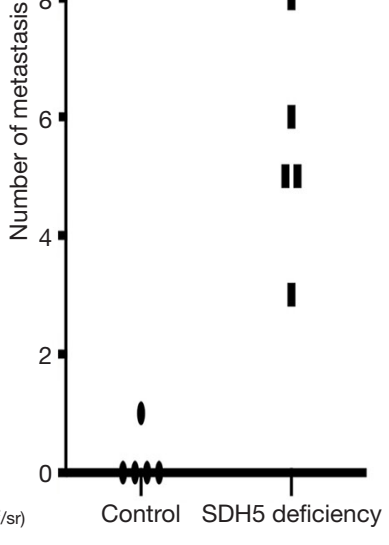

C
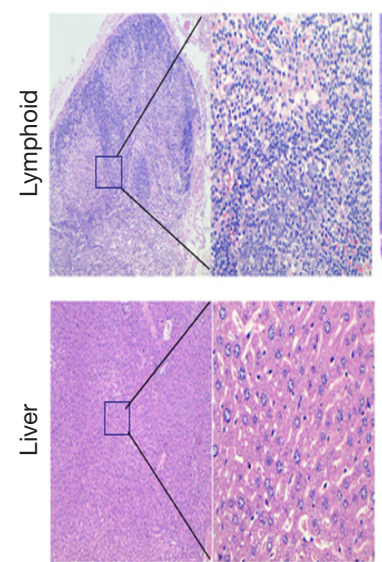
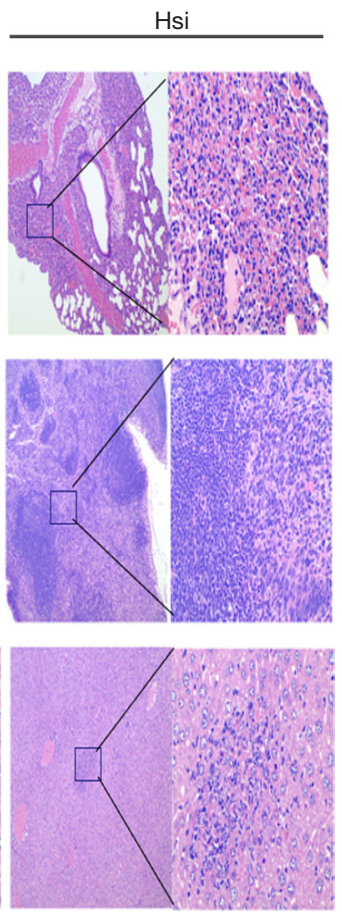

Lymphoid

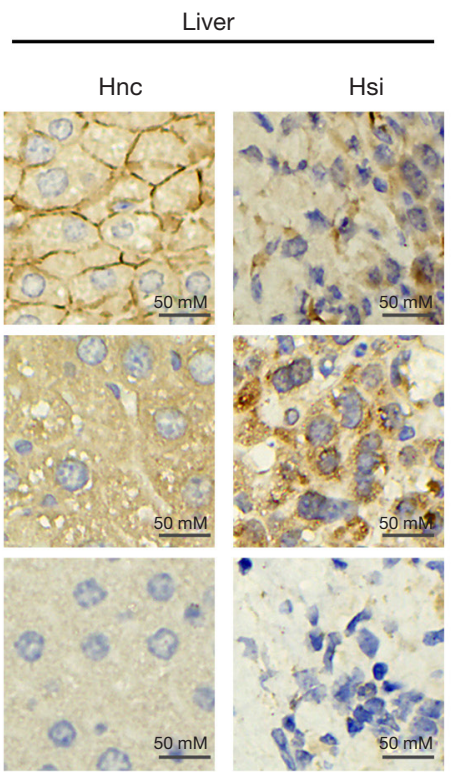

Figure 4 Downregulation of SDH5 enhances tumor metastasis in vivo. (A) Representative BLI images of mice bearing cancer cells (HCC827) with SDH5 Con or KD tumors (n=5); (B) number of metastatic nodules in individual mice at the endpoint; (C) lymph node and liver metastases as assessed by H\&E staining (magnification $\times 100$ ); (D) Vimentin, E-cadherin, and SDH5 staining in the lung, lymph nodes, and liver by IHC. SDH5, succinate dehydrogenase 5; BLI, bioluminescent imaging; IHC, immunohistology.

and drove EMT-related protein changes (Figure 2C). Most importantly, knocking down SDH5 had a superimposed effect that promoted high glucose-induced
EMT (Figure 2D,2E). Furthermore, SDH5-deficient cells exhibited enhanced migration and invasion (Figure $2 F$ ). To verify our previous work (6), we first examined whether 
the GSK3 $\beta-\beta$-Catenin pathway was involved. However, we observed no differences after depleting SDH5, indicating that this signaling pathway was not activated under high glucose conditions (Figure 2H). Cancer cells likely shift among different signaling pathways when they are in different TMEs. Additionally, previous studies have shown that high glucose suppresses GSK3 $\beta-\beta$-Catenin catenin pathway, which may also compensate for the role of SDH5 (20).

Considering that SDH5 is required for an intact Krebs cycle, we found that the loss of SDH5 promoted glycolysis in NSCLC cells. Since HIF-1 $\alpha$ functions as a master regulator in the reprogramming of cell metabolism in favor of glycolysis, we evaluated whether SDH5 promotes glycolysis through HIF-1 $\alpha$. SDH5 deficiency stabilized HIF-1a, which is dependent on ROS production and its target antioxidant enzymes (Figure 3). In vivo, we first established an in-situ tumor model in nude mice with diabetes. SDH5 depletion caused multiple metastases, leading to downregulated E-cadherin and upregulated vimentin, a finding that was consistent with our in vitro data in Figure 2E,2F.

In recent years, the role of immune compartment in cancer has been a hot topic due to the clinical application of immune check blockade. $\mathrm{T}$ cell infiltration in tumor correlates with better patients' survival, however a few proportions of effector $\mathrm{T}$ cells have been found in metastasis lesions. Previous studies have shown tumor enriched in EMT-related genes decreases lymphocytes infiltration while increases production of immunosuppressive cytokines (e.g., IL-10 and TGF- $\beta$ ) as well as expression of immune check point (CTL4, and TIM-3) (18). More importantly, a recent study across 2000 different tumors highlights a strong correlation between EMT and exhausted immune profile (21). From our previous work, we have found SDH5 is negatively correlated with PD-L1 both in vitro and in vivo. SDH5 could reverse the expression of $\mathrm{PD}-\mathrm{L} 1$ in tumor via the GSK3 $\beta / \beta$-catenin/ZEB 1 pathways. Therefore, depleting SDH5 induces PD-L1 expression in cancer cells which inhibit effector $\mathrm{T}$ cell function via PD-1/PD-L1 axis. In addition, in our study we have found SDH5 deficiency promoted glycolysis of cancer cells which also increased lactic acid production and stabilize HIF1a. Therefore, such a harsh microenvironment would also impair $\mathrm{T}$ cell infiltration and function.

\section{Conclusions}

In summary, we demonstrated for the first time that
SDH5 plays a crucial role in regulating cancer cell glucose metabolism and metastasis. SDH5 deficiency regulates ROS levels to stabilize HIF-1 $\alpha$ by inhibiting Nrf2 activation and its target antioxidant enzymes. Subsequently, HIF-1 $\alpha$ may directly induce EMT (Figure S2). Expanding our studies on the immune system in NSCLC patients with diabetes is warranted in future studies. We believe that the plasma SDH5 expression in NSCLC patients with diabetes is an ideal prognostic biomarker to assess the risk of metastasis and prognosis. It is also applicable to monitor cancer progression by multiple blood collection at different time point which may provide more useful information for clinical decision.

\section{Acknowledgments}

Funding: None.

\section{Footnote}

Reporting Checklist: The authors have completed the ARRIVE reporting checklist. Available at https://dx.doi. org/10.21037/jtd-21-1769

Data Sharing Statement: Available at https://dx.doi. org/10.21037/jtd-21-1769

Conflicts of Interest: All authors have completed the ICMJE uniform disclosure form (available at https://dx.doi. org/10.21037/jtd-21-1769). The authors have no conflicts of interest to declare.

Ethical Statement: The authors are accountable for all aspects of the work in ensuring that questions related to the accuracy or integrity of any part of the work are appropriately investigated and resolved. All procedures performed in this study involving human participants were in accordance with the Declaration of Helsinki (as revised in 2013). The study was approved by The Ethical Committee of Huazhong University of Science and Technology and informed consent was taken from all the patients. Animal experiments were performed under a project license (No. SCXK (HUBEI) 2016-0009) granted by Animal Experimentation Ethics Committee of Huazhong University of Science and Technology (HUST, Wuhan, China), in compliance with Hubei Provincial Animal Care and Use Committee guidelines for the care and use of animals.

Open Access Statement: This is an Open Access article 
distributed in accordance with the Creative Commons Attribution-NonCommercial-NoDerivs 4.0 International License (CC BY-NC-ND 4.0), which permits the noncommercial replication and distribution of the article with the strict proviso that no changes or edits are made and the original work is properly cited (including links to both the formal publication through the relevant DOI and the license). See: https://creativecommons.org/ licenses/by-nc-nd/4.0/.

\section{References}

1. Yao L, Liu M, Huang Y, et al. Metformin Use and Lung Cancer Risk in Diabetic Patients: A Systematic Review and Meta-Analysis. Dis Markers 2019;2019:6230162.

2. Zhu L, Cao H, Zhang T, et al. The Effect of Diabetes Mellitus on Lung Cancer Prognosis: A PRISMAcompliant Meta-analysis of Cohort Studies. Medicine (Baltimore) 2016;95:e3528.

3. Bayley JP, Devilee P. Warburg tumours and the mechanisms of mitochondrial tumour suppressor genes. Barking up the right tree? Curr Opin Genet Dev 2010;20:324-9.

4. Kaelin WG Jr. SDH5 mutations and familial paraganglioma: somewhere Warburg is smiling. Cancer Cell 2009;16:180-2.

5. Kunst HP, Rutten MH, de Mönnink JP, et al. SDHAF2 (PGL2-SDH5) and hereditary head and neck paraganglioma. Clin Cancer Res 2011;17:247-54.

6. Liu J, Gao L, Zhang H, et al. Succinate dehydrogenase 5 (SDH5) regulates glycogen synthase kinase $3 \beta-\beta-$ catenin-mediated lung cancer metastasis. J Biol Chem 2013;288:29965-73.

7. Tuo Z, Zong Y, Li J, et al. PD-L1 regulation by SDH5 via $\beta$-catenin/ZEB1 signaling. Oncoimmunology 2019;8:1655361.

8. Dongre A, Weinberg RA. New insights into the mechanisms of epithelial-mesenchymal transition and implications for cancer. Nat Rev Mol Cell Biol 2019;20:69-84.

9. Yang SW, Zhang ZG, Hao YX, et al. HIF-1 $\alpha$ induces the epithelial-mesenchymal transition in gastric cancer stem cells through the Snail pathway. Oncotarget 2017;8:9535-45.

10. Zong Y, Li Q, Zhang F, et al. SDH5 Depletion Enhances Radiosensitivity by Regulating p53: A New Method for Noninvasive Prediction of Radiotherapy Response. Theranostics 2019;9:6380-95.

11. Mariappan MM, Shetty M, Sataranatarajan K, et al.
Glycogen synthase kinase 3 beta is a novel regulator of high glucose- and high insulin-induced extracellular matrix protein synthesis in renal proximal tubular epithelial cells. J Biol Chem 2008;283:30566-75.

12. Lum JJ, Bui T, Gruber M, et al. The transcription factor HIF-1alpha plays a critical role in the growth factordependent regulation of both aerobic and anaerobic glycolysis. Genes Dev 2007;21:1037-49.

13. Asmat U, Abad K, Ismail K. Diabetes mellitus and oxidative stress-A concise review. Saudi Pharm J 2016;24:547-53.

14. Kaneto H, Katakami N, Matsuhisa M, et al. Role of reactive oxygen species in the progression of type 2 diabetes and atherosclerosis. Mediators Inflamm 2010;2010:453892.

15. Lim S, Liu H, Madeira da Silva L, et al. Immunoregulatory Protein B7-H3 Reprograms Glucose Metabolism in Cancer Cells by ROS-Mediated Stabilization of HIF1 $\alpha$. Cancer Res 2016;76:2231-42.

16. Hwang J, Jin J, Jeon S, et al. SOD1 suppresses proinflammatory immune responses by protecting against oxidative stress in colitis. Redox Biol 2020;37:101760.

17. $\mathrm{Ma}$ Q. Role of nrf2 in oxidative stress and toxicity. Annu Rev Pharmacol Toxicol 2013;53:401-26.

18. Melero I, Rouzaut A, Motz GT, et al. T-cell and NKcell infiltration into solid tumors: a key limiting factor for efficacious cancer immunotherapy. Cancer Discov 2014;4:522-6.

19. Mills EL, Kelly B, Logan A, et al. Succinate Dehydrogenase Supports Metabolic Repurposing of Mitochondria to Drive Inflammatory Macrophages. Cell 2016;167:457-470.e13.

20. Ring DB, Johnson KW, Henriksen EJ, et al. Selective glycogen synthase kinase 3 inhibitors potentiate insulin activation of glucose transport and utilization in vitro and in vivo. Diabetes 2003;52:588-95.

21. Mak MP, Tong P, Diao L, et al. A Patient-Derived, PanCancer EMT Signature Identifies Global Molecular Alterations and Immune Target Enrichment Following Epithelial-to-Mesenchymal Transition. Clin Cancer Res 2016;22:609-20.

Cite this article as: Li J, Tuo Z, Zong Y, Liu J. Succinate dehydrogenase 5 regulates lung cancer metastasis by reprogramming glucose metabolism. J Thorac Dis 2021;13(11):6427-6438. doi: 10.21037/jtd-21-1769 
Supplementary

Table S1 Patient characteristics

\begin{tabular}{lccc}
\hline Characteristic & Total $(\mathrm{N}=30)$ & Mean SDH5 mRNA in plasma (range) & Mean SDH5 mRNA in tissue (range) \\
\hline Age, yr, median (range) & $62.4(42-84)$ & $1.81(0.65-3.67)$ & $12.70(3.47-27.75)$ \\
Sex & & & 13.63 \\
Male & 20 & 1.86 & 10.82 \\
Female & 10 & 1.71 & 18.28 \\
Stage & & & 7.1 \\
I & 15 & 2.61 & \\
III & 15 & 1.01 & \\
\hline
\end{tabular}

Table S2 Sequences of genes used in this study

The sequences of the primers are as follows

SDH5-fw (GACTTCGTCGCTGATGCTTG)

SDH5-rv (GTTGGGCTGTCACCTCTGTA),

HIF-1 $\alpha$-fw (GAAAGCGCAAGTCTTCAAAG)

HIF-1 $\alpha$-rv (TGGGTAGGAGATGGAGATGC)

GAPDH-fw (5'-ACCACAGTCCATGCCATCAC-3')

GAPDH-rv (5'-TCCACCACCCTGTTGCTGTA-3') 
A

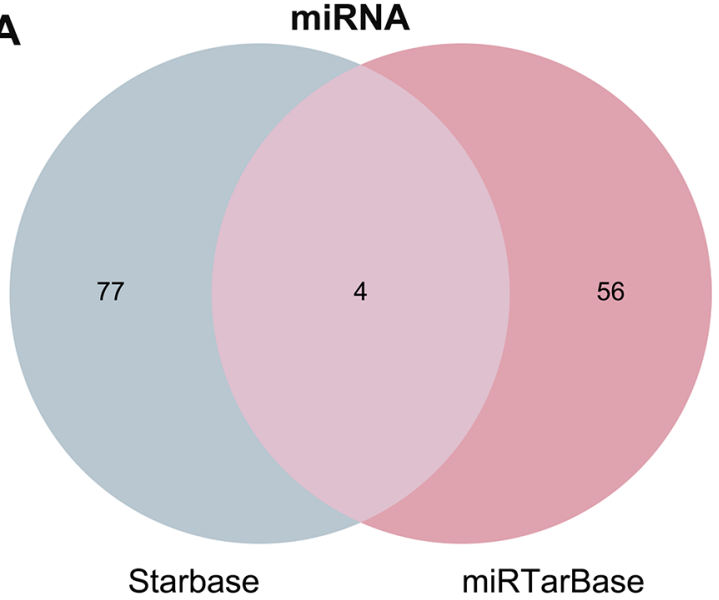

B LncRNA

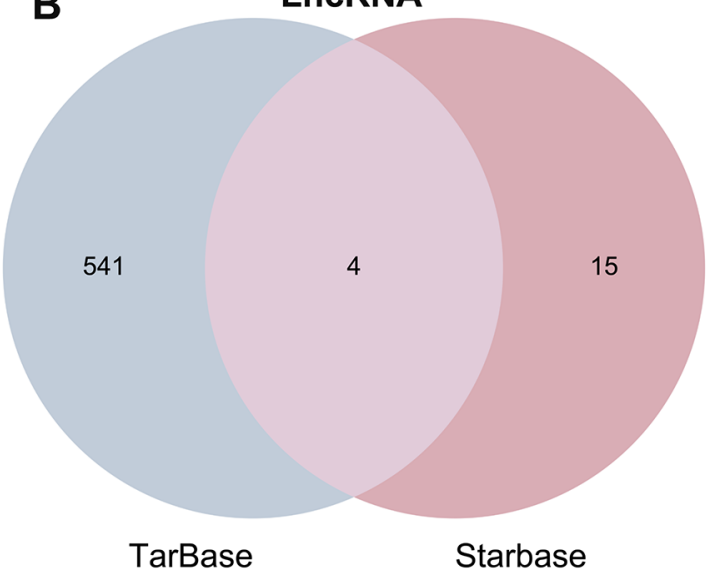

D

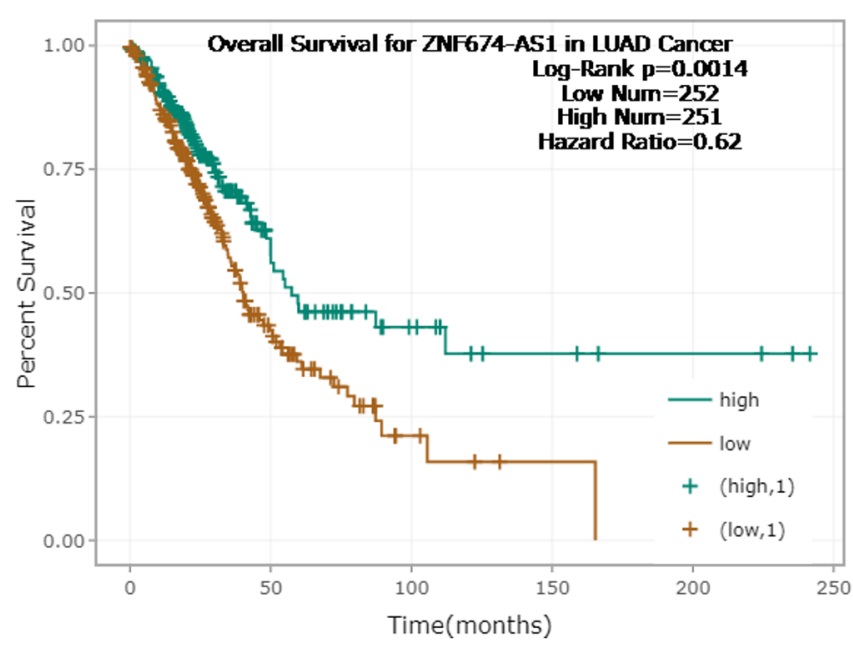

Figure S1 Construction of ceRNA network. (A) Results of miRNA target predicted by mirTarBase and StarBase. (B) Results of lncRNA targets predicted by Tarbase and StarBase. (C) The correlation of ZNF674-AS1 and SDH5 in LUAD. (D) The prognostic value of ZNF674-AS1 in LUAD. 


\section{Cancer cell metabolism in}

SDH5 Deficient conditions

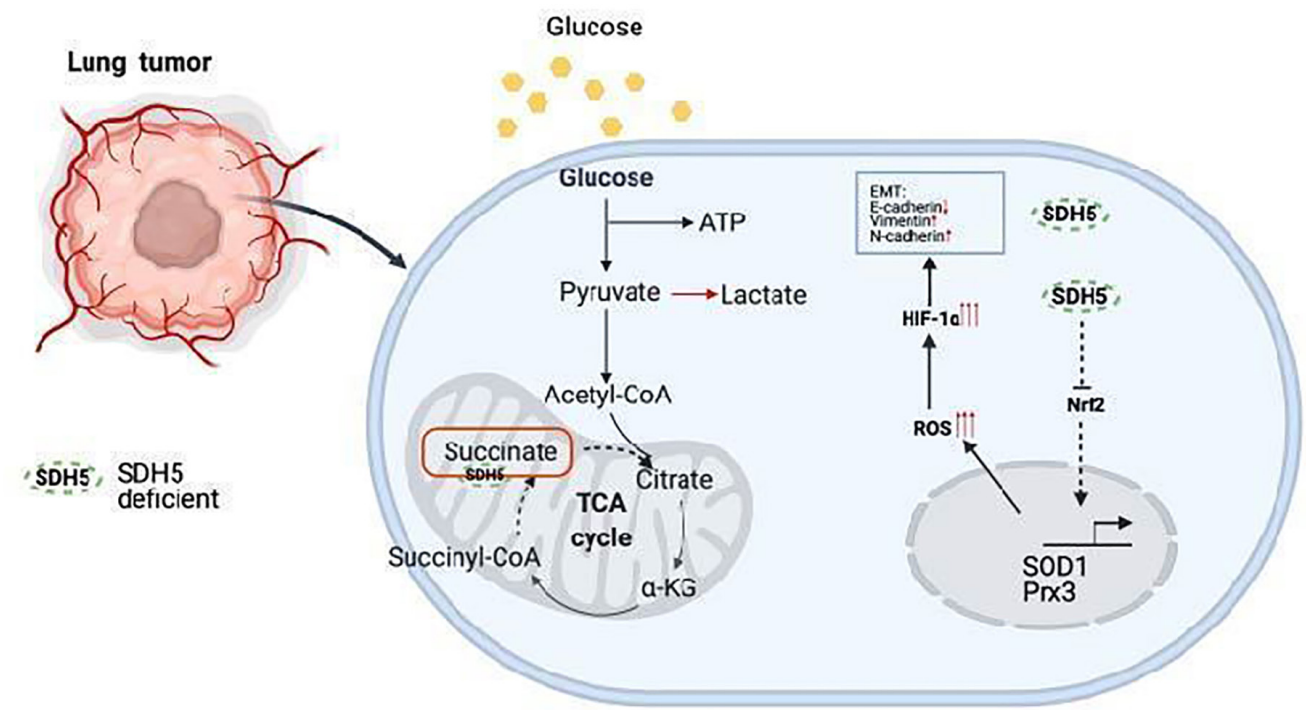

Figure S2 Model of the role of SDH5 in regulating glucose metabolism and metastasis. SDH5 deficiency suppresses Nrf2 nuclear translocation, which reduces transcription of the antioxidant enzymes, SOD1 and PRDX3. The increased ROS in SDH5-deficient cancer cells further stabilize HIF-1 $\alpha$ and promote pyruvate conversion into lactate. Thus, the lactic atmosphere and HIF-1 $\alpha$ promote EMT in cancer cells and tumor metastasis. SDH5, succinate dehydrogenase 5; ROS, reactive oxygen species; HIF-1 $\alpha$, hypoxia-inducible factor 1-alpha; EMT, epithelial-to-mesenchymal transition. 